\title{
Analytical Solutions and Optimization of the Exo-Irreversible Schmidt Cycle with Imperfect Regeneration for the 3 Classical Types of Stirling Engine
}

\author{
P. Rochelle and L. Grosu \\ Laboratoire d'Énergétique, de Mécanique et d'Électromagnétisme, Université Paris Ouest, 50 rue de Sèvres, 92410 Ville d'Avray - France \\ e-mail: pierre.rochelle@u-paris10.fr - lavinia.grosu@u-paris10.fr
}

Résumé - Solutions analytiques et optimisation du cycle de Schmidt irréversible à régénération imparfaite appliquées aux 3 types classiques de moteur Stirling - Le "vieux" moteur Stirling est l'un des moteurs à sources multiples d'énergie les plus prometteurs pour le futur. Des modèles élémentaires simples et réalistes sont utiles pour faciliter l'optimisation de configurations préliminaires du moteur. En plus de nouvelles solutions analytiques qui réduisent fortement le temps de calcul, cette étude du cycle moteur de Schmidt-Stirling modifié est entreprise avec le point de vue de l'ingénieur en introduisant les exo-irréversibilités dues aux transferts thermiques. Les paramètres de référence sont des contraintes technologiques ou physiques : la pression maximum, le volume maximum, les températures de paroi extrêmes et la conductance totale, alors que les paramètres d'optimisation ajustables sont le rapport volumétrique de compression, les rapports de volume mort, le déphasage des volumes balayés, les caractéristiques du gaz, le rapport des conductances "chaude" et "froide" et l'efficacité du régénérateur. Des expressions analytiques nouvelles pour les caractéristiques de fonctionnement du moteur : puissance, travail, rendement, pression moyenne, vitesse maximale, sont établies et quelques nombres de références adimensionnels ou pas sont présentés ainsi que des exemples d'optimisation de la puissance en fonction de la vitesse réduite (adimensionnelle), du rapport des volumes et de l'angle de déphasage.

\footnotetext{
Abstract - Analytical Solutions and Optimization of the Exo-Irreversible Schmidt Cycle with Imperfect Regeneration for the 3 Classical Types of Stirling Engine - The "old" Stirling engine is one of the most promising multi-heat source engines for the future. Simple and realistic basic models are useful to aid in optimizing a preliminary engine configuration. In addition to new proper analytical solutions for regeneration that dramatically reduce computing time, this study of the Schmidt-Stirling engine cycle is carried out from an engineer-friendly viewpoint introducing exo-irreversible heat transfers. The reference parameters are the technological or physical constraints: the maximum pressure, the maximum volume, the extreme wall temperatures and the overall thermal conductance, while the adjustable optimization variables are the volumetric compression ratio, the dead volume ratios, the volume phase-lag, the gas characteristics, the hot-to-cold conductance ratio and the regenerator efficiency. The new normalized analytical expressions for the operating characteristics of the engine: power, work, efficiency, mean pressure, maximum speed of revolution are derived, and some dimensionless and dimensional reference numbers are presented as well as power optimization examples with respect to non-dimensional speed, volume ratio and volume phase-lag angle.analytical solutions.
} 


\section{NOMENCLATURE}

\section{Variables}

$c_{v} \quad$ Specific heat at constant volume $\left(\mathrm{J} \cdot \mathrm{kg}^{-1} \cdot \mathrm{K}^{-1}\right)$

E Energy (J)

$h \quad$ Specific enthalpy $(\mathrm{J} / \mathrm{kg})$

$k \quad$ Regeneration loss factor (-)

$K$ Conductance $\left(\mathrm{W} . \mathrm{K}^{-1}\right)$

$m \quad$ Mass of working gas in ideal cycle $(\mathrm{kg})$

$n \quad$ Speed of revolution (rps)

$p \quad$ Pressure $(\mathrm{Pa})$

$P^{*} \quad$ Normalized mechanical power (-)

$Q \quad$ Heat (J)

$\dot{Q} \quad$ Thermal power $(\mathrm{W})$

$r \quad$ Gas constant $\left(\mathrm{J} \cdot \mathrm{kg}^{-1} \cdot \mathrm{K}^{-1}\right)$

$T$ Temperature (K)

$U \quad$ Internal energy ( $\mathrm{J})$

$V \quad$ Volume $\left(\mathrm{m}^{3}\right)$

$W \quad$ Work $(\mathbf{J})$

$\dot{W} \quad$ Mechanical power $(\mathrm{W})$

\section{Greek symbols}

$\alpha \quad$ Conductance ratio (-)

$\varepsilon \quad$ Volumetric compression ratio (-)

$\gamma \quad$ Adiabatic exponent (-)

$\eta \quad$ Efficiency (-)

$\tau$ Temperature ratio (-)

\section{INTRODUCTION}

To study machine cycles in a more realistic way than basic classical thermodynamics do, one introduces the exo-irreversibilities due to the finite heat transfer rate between the wall source (or sink or regenerator) and the working fluid and, sometimes, those due to internal and/or external frictions and thermal losses. At constant heat conductance, heat flows and work, as well as theoretical power and thermal efficiency, are determined by the temperature gap - the lower the cycle period, the higher the gap; the higher the heat flows, the lower the work. However, often, an increase in heat flows is associated with a decrease of efficiency, thus the point of maximum power is not the point of maximum efficiency. Moreover, in addition to the use of source and sink temperatures $\left(T_{H}\right.$ and $\left.T_{L}\right)$ as obvious given parameters, power optimization is generally carried out using the working gas mass $(m)$ as a reference parameter. For engineers, though, the working gas mass is not the preferred parameter to refer to because practical problems are mainly constrained by technical and physical considerations such as material mechanical- and thermal resistance, bulk volume, and heat exchanger conductance and efficiency. Consequently, it would be desirable to introduce, and substitute for the mass, parameters such as the maximum allowed pressure $\left(p_{\max }\right)$, maximum allowed volume $\left(V_{\max }\right)$, and maximum allowed exchanger area or conductance $\left(K_{T}\right)$. Using speed of revolution instead of time as the main variable is also of prime interest because heat and mass transfers, as well as fluid and mechanical frictions, are directly speed-dependant and thus should be naturally expressed with respect to it. To date, the engineer-friendly finite-time perspective has been given only slight consideration (see the welldocumented study of Durmayaz et al. [1]). In the following sections we develop analytical solutions to show that new conclusions and propositions arise from this more practical approach and that analytical solutions for the exchanged energies lead to a significant improvement in computing time for initial-optimization procedures.

\section{CASE OF ENDO-REVERSIBLE EXO-IRREVERSIBLE IDEAL CARNOT-LIKE CYCLE WITH IMPERFECT REGENERATION}

\subsection{General Case}

This endo-reversible cycle with (Stirling-, Ericsson-, ..., cycles) or without (Carnot cycle) regeneration is assumed to evolve between two reservoirs at constant wall temperatures $T_{H}$ and $T_{L}$ (overall temperature ratio $\tau=T_{L} / T_{H}$ ), with an isothermal heat delivery $Q_{\text {inrev }}$ to the hot gas at temperature $T_{h}$, an isothermal heat release $Q_{\text {outrev }}$ from the cold gas at $T_{l}$ and a delivered work $W$. In case of an endo-reversible cycle with imperfect regeneration, this is revealed by a difference between the inflow and outflow temperatures (resp. specific enthalpies) at each end of the regenerator. To preserve the ideal pressure/temperature history in the swept volumes, the imperfect regeneration must be continuously compensated with an added-heat delivery $\Delta Q_{\text {reg }}$ from the hot source to the gas issuing from the hot outlet of the regenerator into the expansion volume (Fig. 1, 2) to rise the outlet temperature level to the one in the volume.

The same amount of heat is assumed to be lost to the low temperature sink from the gas issuing from the cold outlet of the regenerator into the compression volume. From the endoreversibility assumption, it comes that the ratio of the isothermally transferred heats is equal to the "internal" ratio $\tau_{i}$ of the temperatures of the hot- and cold isothermal volume gases:

$$
\frac{\left|Q_{\text {outrev }}\right|}{Q_{\text {inrev }}}=\frac{T_{l}}{T_{h}}=\tau_{i} .
$$

Hence, the total heat $Q_{i n}$ delivered to the gas in the hot (expansion) volume is the sum of the isothermally delivered heat $Q_{\text {inrev }}$ added to the imperfect-regeneration compensating heat $\Delta Q_{\text {reg }}$ and the total heat $Q_{\text {out }}$ released from the gas in the cold (compression) volume is the sum of the isothermally 


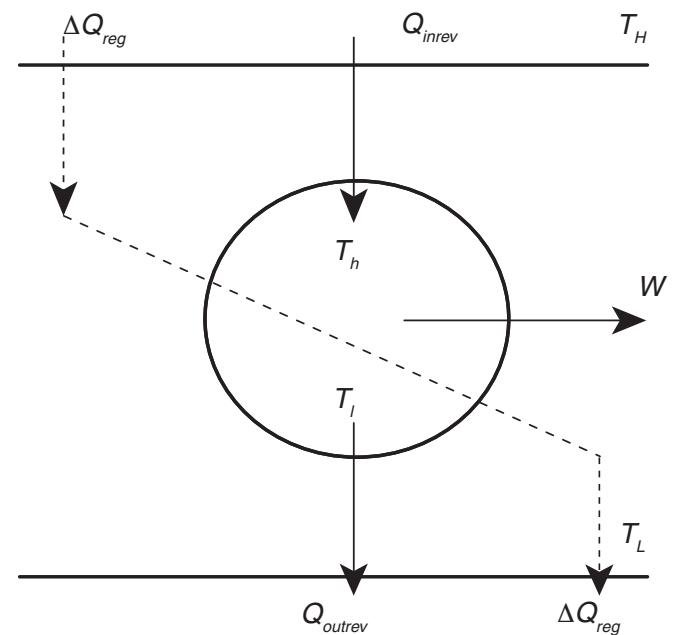

Figure 1

Balance of energy transfers in an endo-reversible exoirreversible cycle with imperfect regeneration.

released heat $Q_{\text {outrev }}$ added to the imperfect-regeneration heat loss $-\Delta Q_{\text {reg }}$ :

$$
\begin{gathered}
Q_{\text {in }}=Q_{\text {inrev }}+\Delta Q_{\text {reg }} \\
Q_{\text {out }}=Q_{\text {outrev }}-\Delta Q_{\text {reg }} .
\end{gathered}
$$

$\Delta Q_{\text {reg }}$ is a part of the heat $Q_{r e g}^{+}$which is reversibly released and caught by the regenerator matrix in the case of perfect regeneration. Let $\eta_{\text {reg }}$ be the regenerator efficiency, then:

$$
\Delta Q_{\text {reg }}=\left(1-\eta_{\text {reg }}\right) \cdot Q_{\text {reg }}^{+} \text {. }
$$

$Q_{\text {reg }}^{+}$and $Q_{\text {inrev }}$ are dependant on the reference pressure, the geometry and kinematics of the engine as well as on the temperature ratio $\tau_{i}$.

The work $W$ is the sum of the delivered heat $(+)$ and released heat (-):

$$
|W|=Q_{\text {in }}+Q_{\text {out }}=Q_{\text {inrev }}+Q_{\text {outrev }}=Q_{\text {inrev }} \cdot\left(1-\tau_{i}\right) .
$$

The internal Carnot efficiency $\eta_{i}$ is the cycle efficiency in the case of perfect regeneration:

$$
\eta_{i}=\frac{|W|}{Q_{\text {inrev }}}=1-\tau_{i} .
$$

In case of exo-reversibility (classical thermodynamics), and $\tau_{i}=\tau$ and $\eta_{i}=\eta_{\text {Carnot }}=1-\tau$.

\subsection{Effect of Exo-Irreversibility and Imperfect Regeneration}

Assuming $K_{T}$ as the total convective heat conductance of the gas, which is the sum of the cycle time-averaged hot and cold wall/fluid conductances, $\alpha$ as the relative part of conductance involved in the heat transfer at hot source and $n$ as the speed of revolution. Hence, energies could be written as follows:

$$
\begin{gathered}
Q_{\text {in }}=\alpha \cdot \frac{K_{T}}{n} \cdot\left(T_{H}-T_{h}\right)=\alpha \cdot \frac{K_{T} \cdot T_{H}}{n} \cdot\left(1-\tau_{h}\right) \\
Q_{\text {out }}=(1-\alpha) \cdot \frac{K_{T}}{n} \cdot\left(T_{L}-T_{l}\right)=(1-\alpha) \cdot \frac{K_{T} \cdot T_{H}}{n} \cdot\left(\frac{T_{L}}{T_{H}}-\frac{T_{l}}{T_{H}}\right)_{(8)} \\
=(1-\alpha) \cdot \frac{K_{T} \cdot T_{H}}{n} \cdot\left(\tau-\tau_{l}\right) \\
|W|=Q_{o u t}+Q_{\text {in }}=\frac{K_{T} \cdot T_{H}}{n} \cdot\left[\alpha \cdot\left(1-\tau_{h}\right)+(1-\alpha) \cdot\left(\tau-\tau_{l}\right)\right] \\
=\frac{K_{T} \cdot T_{H}}{n} \cdot\left\{[\alpha+(1-\alpha) \cdot \tau]-\tau_{h} \cdot\left[\alpha+(1-\alpha) \cdot \tau_{i}\right]\right\}
\end{gathered}
$$

and, noting that $\tau_{l}=\tau_{h} \cdot \tau_{i}$, it gives, first with Equations (5) and (9), second with Equations (2), (4), (7) and (8):

$$
\tau_{h}=\frac{[\alpha+(1-\alpha) \cdot \tau]-\frac{n \cdot Q_{\text {inrev }} \cdot\left(1-\tau_{i}\right)}{K_{T} \cdot T_{H}}}{\left[\alpha+(1-\alpha) \cdot \tau_{i}\right]}
$$

and

$$
\tau_{h}=1-\frac{n \cdot Q_{\text {inrev }}}{\alpha \cdot K_{T} \cdot T_{H}} \cdot\left[1+\left(1-\eta_{\text {reg }}\right) \cdot \frac{Q_{\text {reg }}^{+}}{Q_{\text {inrev }}}\right] .
$$

Combining Equations (10a) and (10b) and assuming no explicit dependence of the various parameters on $n$ excepted the one given by (11):

$$
n=\frac{K_{T} \cdot T_{H}}{Q_{\text {inrev }}} \cdot \frac{\alpha \cdot(1-\alpha) \cdot\left(\tau_{i}-\tau\right)}{\left\{\tau_{i}+\left[\alpha+\tau_{i} \cdot(1-\alpha)\right] \cdot\left(1-\eta_{\text {reg }}\right) \cdot \frac{Q_{\text {reg }}^{+}}{Q_{\text {inrev }}}\right\}}
$$

where $\frac{K_{T} \cdot T_{H}}{Q_{\text {inrev }}}$ has dimension of a speed of revolution (in rps) or an inverse of time (in $1 / \mathrm{s}$ ).

The expression of $n$ (Eq. 11) could be re-introduced into Equation (10) to get $\tau_{h}$ with respect to $\tau_{i}$ and then into Equation (6) to get $Q_{i n}$.

The cycle efficiency is:

$$
\eta=\frac{|W|}{Q_{i n}}=\frac{\left(1-\tau_{i}\right)}{1+\left(1-\eta_{\text {reg }}\right) \cdot \frac{Q_{\text {reg }}^{+}}{Q_{\text {inrev }}}}
$$

and, from Equations (5) and (11), with respect to $\tau_{i}$ the power is:

$$
P=n \cdot|W|=K_{T} \cdot T_{H} \cdot \frac{\alpha \cdot(1-\alpha) \cdot\left(\tau_{i}-\tau\right) \cdot\left(1-\tau_{i}\right)}{\left\{\tau_{i}+\left[\alpha+\tau_{i} \cdot(1-\alpha)\right] \cdot\left(1-\eta_{\text {reg }}\right) \cdot \frac{Q_{\text {reg }}^{+}}{Q_{\text {irrev }}}\right\}} .
$$


With a given $K_{T} \cdot T_{H}$, derivations of Equation (13) and equalization to zero of the derivatives give the deduced optimum values $\eta_{\text {reg }}=1, \alpha=0.5$ (Feidt et al. [2]), $\tau_{i}=\sqrt{\tau}$ and, then, the (overall) maximum maximorum power:

$$
P_{\text {max } \max }=K_{T} \cdot T_{H} \cdot \frac{(1-\sqrt{\tau})^{2}}{4} .
$$

$\tau_{i}$ evolves from $\tau$ at very low speed, to $1\left(T_{h}=T_{\nu}\right)$, at the speed limit $n_{\lim }$. At the speed limit, with the same assumption as for Equation (11) and, as an addition, with the obvious assumption of no heat transfer for regeneration-loss compensation at $T_{h}=T_{l}$, then $Q_{\text {reg }}^{+}=0$ and $Q_{\text {inrev }}\left(\tau_{i}\right)=Q_{\text {inrev }}(1)=$ $Q_{\text {inrev1 }}$, giving:

$$
n_{\lim }=\frac{K_{T} \cdot T_{H}}{Q_{\text {inrev } 1}} \cdot \alpha \cdot(1-\alpha) \cdot(1-\tau)
$$

It gives also:

$$
\tau_{h \lim }=\tau_{\lim }=\alpha+(1-\alpha) \cdot \tau
$$

and, using $\alpha$ optimum value:

$$
\begin{gathered}
n_{\lim \min }=\frac{K_{T} \cdot T_{H}}{Q_{\text {inrev } 1}} \cdot \frac{(1-\tau)}{4} \\
\dot{Q}_{i n \lim \min }=K_{T} \cdot T_{H} \cdot \frac{(1-\tau)}{4} .
\end{gathered}
$$

\section{THE SCHMIDT-STIRLING EXO-IRREVERSIBLE ENDO-REVERSIBLE CYCLE WITH IMPERFECT REGENERATION}

$Q_{\text {reg }}^{+}$and $Q_{\text {inrev }}$ depend on the type of reversible cycle. Let us examine the case of the Schmidt-Stirling cycle.

There are 3 basic configurations for the classical Stirling engine (Fig. 2). A classical way to model this engine with some realism is to use the Schmidt model. Its main assumptions, slightly completed, are:

- same instantaneous pressure throughout the engine;

- use of an ideal gas as the working fluid;

- constant working fluid mass (no leakage, no delivery) during a cycle;

- constant cylinder wall temperature;

- harmonic/sinusoidal movement of the pistons (idealized crankshaft);

- constant temperature of gas in the hot and cold volumes. This is nearly verified in LTD (Low Temperature Differential) Stirling machines with a low speed of revolution and heat exchanging cylinder head and wall;

- constant speed of revolution;

- perfect regeneration.
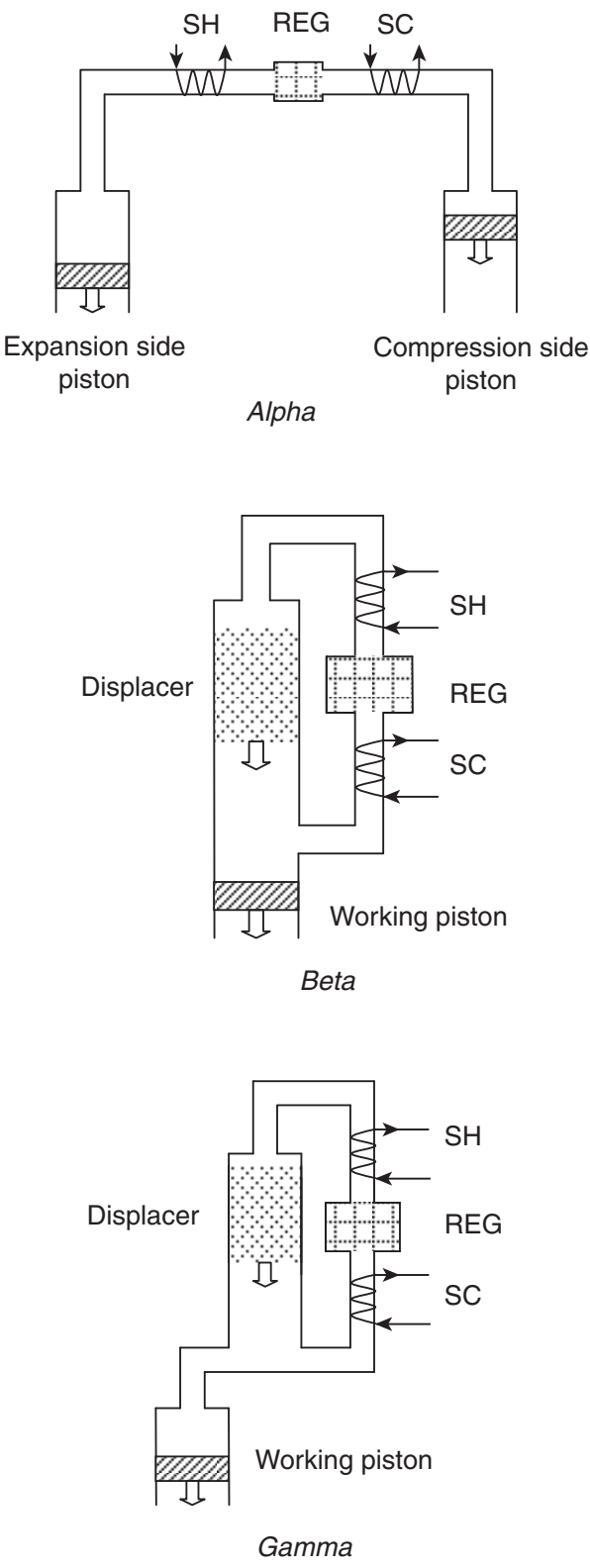

Figure 2

The 3 classical types of Stirling engine configuration.

This last assumption implies that the entirety of the heat released to the regenerator material during the gas flow from the hot volume to the cold volume is reversibly restituted to the gas during the back flow, at the same levels of temperature. In our case of imperfect regeneration, it will be assumed that the gas pressure/temperature history will remain the same but the part of regeneration heat lost (to the cold sink, by conduction or other transport) will be continuously compensated by a supplement of heat $\Delta Q_{\text {reg }}$ provided by the hot source during each cycle as seen before (Sect. 1.1). 


\subsection{Instantaneous Volume Expressions}

In the 3 types of engine, the expansion (hot) space variation has a unique expression:

$$
V_{E}=\frac{V_{E 0}}{2} \cdot[1-\cos (\varphi)]
$$

where $\varphi$ is the rotation angle of the idealized crankshaft and $V_{E 0}$ is the "swept expansion volume"; in the case of beta- and gamma-type engines, this is the displacer swept volume.

For the compression space, in the case of beta- or gammatype engines, that geometrically differ by either a not commonor a common cylinder and an overlapping swept volume), there is a combination of volume variations; it could be expressed as:

$V_{C}=a_{j} \cdot \frac{V_{E 0}}{2} \cdot[1+\cos (\varphi)]+\frac{V_{C 0}}{2} \cdot\left[1-\cos \left(\varphi-\varphi_{0}\right)\right]-b_{j} \cdot V_{o l}$

where $a_{j}$ and $b_{j}$ values, displayed in Table 1 , depend on the type of engine, $\varphi_{0}$ is the phase lag angle of the piston movements and $V_{C 0}$ is the "swept compression volume". In fact, in our case of beta- and gamma-type engines, it only is the working-piston swept volume. $V_{o l}$ is the overlapping volume in the case of a beta-type engine and is due to the intrusion of the displacer piston into the working piston swept volume. Here, this volume equation is obtained by assuming there is one and only one contact point between the displacer piston and the working piston $\left(V_{C}=0\right.$ and $\frac{\partial V_{C}}{\partial \varphi}=0$ for $\left.\varphi=\varphi_{\text {contact }}\right)$, during
their cyclical movement.

\section{TABLE 1}

\begin{tabular}{c|c|c|c}
\hline Engine-type & Alpha & Beta & Gamma \\
\hline$a_{j}$ & 0 & 1 & 1 \\
\hline$b_{j}$ & 0 & 1 & 0 \\
\hline
\end{tabular}

Dead volumes, due to the heat exchangers and the imperfect geometry of the cylinder volumes must be taken into account. Let $V_{E S}, V_{C S}, V_{R}$ be the 3 dead volumes respectively related to the expansion and hot exchanger volumes $\left(V_{E S}\right)$, to the compression and cold exchanger volumes $\left(V_{C S}\right)$ and to the regenerator volume $\left(V_{R}\right)$; the sum of these will be the total dead volume $V_{S}$.

The total instantaneous working gas volume $V_{t}$ is the sum of the previous ones:

$$
\begin{aligned}
V_{t}= & V_{E}+V_{C}+V_{S}=\frac{V_{E 0}}{2} \cdot\left\{[1-\cos (\varphi)]+a_{j} \cdot[1+\cos (\varphi)]\right\} \\
& +\frac{V_{C 0}}{2} \cdot\left[1-\cos \left(\varphi-\varphi_{0}\right)\right]-b_{j} \cdot V_{o l}+V_{S} .
\end{aligned}
$$

The maximum global volume is:

$$
V_{T}=V_{E 0}+V_{C 0}+V_{S}-b_{j} V_{o l}
$$

Normalizing the volumes with respect to $V_{T}, V_{t}$ is expressed as a function of expansion-, compression-, deadand overlapping volume ratios $\left(\varepsilon_{E}, \varepsilon_{C}, \varepsilon_{S}\right.$ and $\left.\varepsilon_{o l}\right)$ and, even, of $\omega$ which is the compression to expansion swept volume ratio $\left(\omega=\frac{V_{C 0}}{V_{E 0}}=\frac{\omega_{C}}{\omega_{E}}\right)$ :

$$
\begin{aligned}
V_{t}^{*} & =V_{E}^{*}+V_{C}^{*}+V_{S}^{*} \\
& =\frac{\varepsilon_{E}}{2} \cdot\left\{[1-\cos (\varphi)]+a_{j} \cdot[1+\cos (\varphi)]\right\} \\
& +\frac{\varepsilon_{C}}{2} \cdot\left[1-\cos \left(\varphi-\varphi_{0}\right)\right]-b_{j} \cdot \varepsilon_{o l}+\varepsilon_{S}
\end{aligned}
$$

Using a classical trigonometric relation (see set of $E q$. Al in Appendix) gives:

$$
V_{t}^{*}=B_{V}-A_{V} \cdot \cos \left(\varphi-\varphi_{V}\right)
$$

with $B_{V}, A_{V}$ and $\varphi_{V}$ expressed as:

$$
\left\{\begin{array}{l}
B_{V}=\frac{\varepsilon_{E}}{2} \cdot\left[1+a_{j}+\omega+2 \cdot \frac{\varepsilon_{S}}{\varepsilon_{E}}-2 \cdot b_{j} \cdot \frac{\varepsilon_{o l}}{\varepsilon_{E}}\right] \\
A_{V}=\frac{\varepsilon_{E}}{2} \cdot \sqrt{\left[1-a_{j}+\omega \cdot \cos \left(\varphi_{0}\right)\right]^{2}+\left[\omega \cdot \sin \left(\varphi_{0}\right)\right]^{2}} \\
\sin \left(\varphi_{V}\right)=\frac{\varepsilon_{E}}{2 \cdot A_{V}} \cdot \omega \cdot \sin \left(\varphi_{0}\right) \text { and } \\
\cos \left(\varphi_{V}\right)=\frac{\varepsilon_{E}}{2 \cdot A_{V}} \cdot\left[1-a_{j}+\omega \cdot \cos \left(\varphi_{0}\right)\right]
\end{array}\right.
$$

Let $\delta_{V}=\frac{A_{V}}{B_{V}}$, then one gets the normalized volume $V_{t}^{*}=B_{V} \cdot\left[1-\delta_{V} \cdot \cos \left(\varphi-\varphi_{V}\right)\right]$ and the volumetric compression ratio:

$$
\varepsilon=\frac{V_{t \max }}{V_{t \min }}=\frac{V_{t \max }^{*}}{V_{t \min }^{*}}=\frac{1+\delta_{V}}{1-\delta_{V}} \leftrightarrow \delta_{V}=\frac{\varepsilon-1}{\varepsilon+1} .
$$

\subsection{Instantaneous Pressure Expression}

Assuming a constant working gas mass in the engine, which is the sum of the masses in each volume, this total mass of gas is expressed as a function of the instantaneous pressure and volumes:

$$
m_{T}=\frac{p \cdot V_{E}}{r \cdot T_{h}}+\frac{p \cdot V_{E S}}{r \cdot T_{h}}+\frac{p \cdot V_{C}}{r \cdot T_{l}}+\frac{p \cdot V_{C S}}{r \cdot T_{l}}+\frac{p \cdot V_{R}}{r \cdot T_{R}}
$$
where, remembering that $\tau_{i}=\frac{T_{l}}{T_{h}}$, the regenerator mean
temperature could be either:

$$
T_{R}=\frac{T_{h}+T_{l}}{2}=T_{h} \cdot \frac{1+\tau_{i}}{2}
$$


or, assuming a linear temperature profile in the regenerator as Urieli and Berchowitz [3] did:

$$
T_{R}=\frac{T_{h}-T_{l}}{\ln \left(\frac{T_{h}}{T_{l}}\right)}=T_{h} \cdot \frac{\left(1-\tau_{i}\right)}{\ln \left(\frac{1}{\tau_{i}}\right)},
$$

then $\frac{T_{h}}{T_{R}}=\frac{2}{1+\tau_{i}}$ or $\frac{T_{h}}{T_{R}}=\frac{-\ln \left(\tau_{i}\right)}{1-\tau_{i}}$. For $p$, Equation (26) gives:

$$
\begin{aligned}
p= & \frac{m_{T} \cdot r}{\frac{V_{E}+V_{E S}}{T_{h}}+\frac{V_{C}+V_{C S}}{T_{l}}+\frac{V_{R}}{T_{R}}} \\
& =\frac{m_{T} \cdot r \cdot T_{h}}{\left(V_{E}+V_{E S}\right)+\frac{T_{h}}{T_{l}} \cdot\left(V_{C}+V_{C S}\right)+\frac{T_{h}}{T_{R}} \cdot V_{R}}=\frac{N}{D}
\end{aligned}
$$

(see Eq.A2).

After passing $T_{h}$ into the numerator and normalizing the volumes with respect to $V_{T}$, with the same trigonometric method as before, the new denominator $D(E q$. A3), having dimension of a volume, could be written as:

$$
V_{T} \cdot\left[B_{p}-A_{p} \cdot \cos \left(\varphi-\varphi_{p}\right)\right]
$$

with the set of equations obtained by identification of the parameters:

$$
\left\{\begin{array}{l}
B_{p}=\frac{\varepsilon_{E}}{2} \cdot\left[\left(1+\frac{a_{j}}{\tau_{i}}\right)+\frac{\omega}{\tau_{i}}+\frac{2 \cdot \varepsilon_{E S}}{\varepsilon_{E}}+\frac{\left(\varepsilon_{C S}-b_{j} \cdot \varepsilon_{o l}\right)}{\varepsilon_{E}} \cdot \frac{2}{\tau_{i}}+\frac{2 \cdot \varepsilon_{R}}{\varepsilon_{E}} \cdot \frac{T_{h}}{T_{R}}\right. \\
A_{p}=\frac{\varepsilon_{E}}{2} \sqrt{\left[1-\frac{a_{j}}{\tau_{i}}+\frac{\omega}{\tau_{i}} \cos \left(\varphi_{0}\right)\right]^{2}+\left[\frac{\omega}{\tau_{i}} \sin \left(\varphi_{0}\right)\right]^{2}} \\
\sin \left(\varphi_{p}\right)=\frac{\varepsilon_{E}}{2 \cdot A_{p}} \cdot \frac{\omega}{\tau_{i}} \cdot \sin \left(\varphi_{0}\right) \\
\cos \left(\varphi_{p}\right)=\frac{\varepsilon_{E}}{2 \cdot A_{p}}\left[\left(1-\frac{a_{j}}{\tau_{i}}\right)+\frac{\omega}{\tau_{i}} \cdot \cos \left(\varphi_{0}\right)\right] .
\end{array}\right.
$$

The maximum pressure is obtained for $\varphi=\varphi_{p}$ :

$$
p_{\max }=\frac{m_{T} \cdot r \cdot T_{h}}{V_{T} \cdot\left[B_{p}-A_{p}\right]} .
$$

Normalizing $p$ with respect to $p_{\max }$, it becomes:

$$
p^{*}=\frac{1-\delta_{p}}{1-\delta_{p} \cdot \cos \left(\varphi-\varphi_{p}\right)}
$$

where $\delta_{p}=\frac{A_{p}}{B_{p}}$. The maximum to minimum pressure ratio $\varpi$ is:

$$
\varpi=\frac{p_{\max }}{p_{\min }}=\frac{B_{p}+A_{p}}{B_{p}-A_{p}}=\frac{1+\delta_{p}}{1-\delta_{p}} .
$$

\subsection{Expressions of the Work and Isothermally Delivered Heat}

Since the heat $Q_{\text {inrev }}$ delivered isothermally at hot temperature $T_{h}$ during a complete cycle equals the opposite value of the gas work done in the expansion volume $V_{E}$, therefore (with the "continental" convention that the work, as well as the heat, produced and lost by the gas is negative):

$$
\begin{aligned}
& |W|=\left(1-\tau_{i}\right) \cdot Q_{\text {inrev }} \text {, where: } \\
& Q_{\text {inrev }}=-W_{E}=\oint p \cdot d V_{E}=p_{\max } \cdot V_{T} \cdot \oint p^{*} \cdot d V_{E}^{*} \\
& =p_{\max } \cdot V_{T} \cdot\left(1-\delta_{p}\right) \cdot \frac{\varepsilon_{E}}{2} \cdot \oint \frac{\sin (\varphi) \cdot d \varphi}{1-\delta_{p} \cdot \cos \left(\varphi-\varphi_{p}\right)} .
\end{aligned}
$$

It gives, following Meijer [4], Finkelstein [5], Walker [6], Rochelle and Andrzjewski [7] (pp. 745-746) and applying the properties of the finite trigonometric integrals (Dwight [8]) (Eq. A4):

$$
Q_{\text {inrev }}=p_{\max } \cdot V_{T} \cdot \pi \cdot \varepsilon_{E} \frac{\left(1-\delta_{p}\right)}{\delta_{p}} \cdot\left[\frac{1}{\sqrt{1-\delta_{p}^{2}}}-1\right] \cdot \sin \left(\varphi_{p}\right)
$$

and, from the endo-reversibility assumption: $Q_{\text {ourev }}=-\tau_{i} \cdot Q_{\text {inrev }}$ then:

$$
|W|=p_{\max } \cdot V_{T} \cdot\left(1-\tau_{i}\right) \cdot \pi \cdot \varepsilon_{E} \frac{\left(1-\delta_{p}\right)}{\delta_{p}} \cdot\left[\frac{1}{\sqrt{1-\delta_{p}^{2}}}-1\right] \cdot \sin \left(\varphi_{p}\right)
$$

the same ones are given under their normalized form with respect to $p_{\max } \cdot V_{T}$, as follows:

$$
\begin{gathered}
Q_{\text {inrev }}^{*}=\pi \cdot \varepsilon_{E} \frac{\left(1-\delta_{p}\right)}{\delta_{p}} \cdot\left[\frac{1}{\sqrt{1-\delta_{p}^{2}}}-1\right] \cdot \sin \left(\varphi_{p}\right) \\
\text { and }\left|W^{*}\right|=\left(1-\tau_{i}\right) \cdot \pi \cdot \varepsilon_{E} \frac{\left(1-\delta_{p}\right)}{\delta_{p}} \cdot\left[\frac{1}{\sqrt{1-\delta_{p}^{2}}}-1\right] \cdot \sin \left(\varphi_{p}\right) .
\end{gathered}
$$

\subsection{Analytical Expression of the Perfect-Regeneration Heat}

In case of perfect regeneration, the gas temperature at each extremity of the regenerator equals the gas temperature in the adjacent volume; hence, the elementary energy balance in the constant-volume regenerator is given by the following equation (developed in Eq.A5):

$d Q_{\text {reg }}=d U_{R}-\sum_{j} h_{j} \cdot d m_{j}=\frac{V_{R} \cdot d p}{\gamma-1}+\frac{\gamma \cdot r}{\gamma-1} \cdot\left(T_{h} \cdot d m_{E}+T_{l} \cdot d m_{C}\right)$ the elementary masses $d m_{E}$ and $d m_{C}$ being considered as positive when issuing from the regenerator. From the isothermal assumption in the adjacent volumes:

$d m_{E}=\frac{d\left(p \cdot V_{E}^{+}\right)}{r \cdot T_{h}}$ and $d m_{C}=\frac{d\left(p \cdot V_{C}^{+}\right)}{r \cdot T_{l}}$

where $V_{E}^{+}=V_{E}+V_{E S}$ and $V_{C}^{+}=V_{C}+V_{C S}$.

Noting that $V_{E}^{+}+V_{C}^{+}+\frac{V_{R}}{\gamma}=V_{t}-\frac{\gamma-1}{\gamma} \cdot V_{R}=V_{t R}$ 
$d Q_{\text {reg }}$ can be rewritten as:

$$
d Q_{\text {reg }}=\frac{\gamma}{\gamma-1} \cdot\left\{p \cdot d V_{t R}+V_{t R} \cdot d p\right\}=\frac{\gamma}{\gamma-1} \cdot d\left(p \cdot V_{t R}\right)
$$

with $V_{t R}$ used as a provisional volume for the demonstration purpose. Developed under its normalized form and letting

$$
\begin{gathered}
B_{V R}=B_{V}-\frac{\gamma-1}{\gamma} \cdot \varepsilon_{R} \text { and } \delta_{V R}=\frac{A_{V}}{B_{V R}} \text {, it gives } \\
V_{t R}^{*}=B_{V}-\frac{\gamma-1}{\gamma} \cdot \varepsilon_{R}-A_{V} \cdot \cos \left(\varphi-\varphi_{V}\right) \\
=B_{V R} \cdot\left[1-\delta_{V R} \cdot \cos \left(\varphi-\varphi_{V}\right)\right]
\end{gathered}
$$

and it leads to the normalized form of $d Q_{\text {reg }}$ :

$$
\begin{aligned}
d Q_{\text {reg }}^{*} & =\frac{\gamma}{\gamma-1} \cdot d\left(p^{*} \cdot V_{t R}^{*}\right) \\
& =\frac{\gamma \cdot\left(1-\delta_{p}\right)}{(\gamma-1)} \cdot B_{V R} \cdot d\left[\frac{1-\delta_{V R} \cdot \cos \left(\varphi-\varphi_{V}\right)}{1-\delta_{p} \cdot \cos \left(\varphi-\varphi_{p}\right)}\right] .
\end{aligned}
$$

The perfect regeneration heat $Q_{\text {reg }}$ is null on a complete cycle, resulting from the sum of 2 equal and opposed parts. The angles corresponding to the change of sign $d Q_{\text {reg }}$ or $d Q_{\text {reg }}^{*}$ are obtained for $d\left(p^{*} \cdot V_{t R}^{*}\right)=0$ hence, in this case, (from $\mathrm{Eq} . \mathrm{A6})$ :

$$
\begin{aligned}
& {\left[1-\delta_{p} \cdot \cos (\theta)\right] \cdot A_{V} \sin (\theta-\Phi)} \\
& \quad-\left[B_{V R}-A_{V} \cdot \cos (\theta-\Phi)\right] \cdot \delta_{p} \cdot \sin (\theta)=0
\end{aligned}
$$

where $\theta=\varphi-\varphi_{p}$ and $\Phi=\varphi_{V}-\varphi_{p}(=\mathrm{cst})$.

From Equation (32b), $Q_{\text {reg }}^{+*}$ is given by the expression of the definite integral:

$$
\begin{aligned}
Q_{\text {reg }}^{+*} & =\frac{\gamma \cdot\left(1-\delta_{p}\right)}{(\gamma-1)} \cdot B_{V R} \\
\cdot & \cdot\left[\frac{1-\delta_{V R} \cdot \cos \left(\theta_{2}-\Phi\right)}{1-\delta_{p} \cdot \cos \left(\theta_{2}\right)}-\frac{1-\delta_{V R} \cdot \cos \left(\theta_{1}-\Phi\right)}{1-\delta_{p} \cdot \cos \left(\theta_{1}\right)}\right] .
\end{aligned}
$$

The 2 solutions of Equation (33) are obtained, after its decomposition (set of $E q . A 7$ and A8) and the use of the previous trigonometric method, as:

$$
\cos \left(\theta_{i}\right)_{i=1 \text { or } 2}=\frac{\delta_{V R}}{\delta^{\prime} \cdot A^{\prime}} \cdot\left\{\sin (\Phi) \mp \sqrt{\delta^{\prime 2}-1} \cdot\left[\cos (\Phi)-\frac{\delta_{p}}{\delta_{V R}}\right]\right\}
$$

where:

$$
\begin{aligned}
& A^{\prime}=\sqrt{\left[\delta_{p}-\delta_{V R} \cdot \cos (\Phi)\right]^{2}+\left[\delta_{V R} \cdot \sin (\Phi)\right]^{2}} \\
& \text { and } \delta^{\prime}=\frac{A^{\prime}}{\delta_{V R} \cdot \delta_{p} \cdot \sin (\Phi)}
\end{aligned}
$$

Then, after further similar developments, we get Equation (36):

$$
\cos \left(\theta_{i}-\Phi\right)_{i=1 \mathrm{or} 2}=\frac{\delta_{p}}{\delta^{\prime} \cdot A^{\prime}} \cdot\left\{\sin (\Phi) \mp \sqrt{\delta^{\prime 2}-1} \cdot\left[\frac{\delta_{V R}}{\delta_{p}}-\cos (\Phi)\right]\right\} .
$$

Hence, after using Equations (35) and (36) in (34) (see Eq. A9), and after further simplifications and factorizations (see $E q$. A10), we get:

$$
\begin{aligned}
Q_{\text {reg }}^{+*} & =\frac{2 \gamma}{(\gamma-1)} \cdot \frac{B_{V R}}{\left(1+\delta_{p}\right)} \\
& \cdot \sqrt{\left[\delta_{p} \cdot \delta_{V R} \cdot \cos \left(\varphi_{V}-\varphi_{p}\right)-1\right]^{2}-\left(\delta_{p}^{2}-1\right) \cdot\left(\delta_{V R}^{2}-1\right)} .
\end{aligned}
$$

With this result, efficiency $\eta(E q .12)$ and power $P(E q .13)$ could be expressed:

- with the ratio of the developed expressions of isothermally delivered heat $Q_{\text {inrev }}$ and positive exchanged heat of regeneration $Q_{\text {reg }}^{+}$or;

- with the ratio of their normalized expressions (Eq. 30b and 37) given by Equation (40) (see Eq. A11). They are, under there completely developed form, functions of seven parameters $\left(\varepsilon_{E}, \varepsilon_{C}, \varepsilon_{E S}, \varepsilon_{C S}, \varepsilon_{R}, \gamma, \varphi_{0}\right)$ and one variable $\left(\tau_{i}\right)$.

\subsection{Speed, Power and Efficiency Expressions}

Introducing a reference speed of revolution $n_{r e f}=\frac{K_{T} \cdot T_{H}}{p_{\max } \cdot V_{T}}$ into Equation (11), the normalized speed is given by:

$$
n^{*}=\frac{1}{Q_{\text {inrev }}^{*}} \cdot \frac{\alpha \cdot(1-\alpha) \cdot\left(\tau_{i}-\tau\right)}{\left\{\tau_{i}+\left[\alpha+\tau_{i} \cdot(1-\alpha)\right] \cdot\left(1-\eta_{\text {reg }}\right) \cdot \frac{Q_{\text {reg }}^{+*}}{Q_{\text {inrev }}^{*}}\right\}}
$$

the normalized power is:

$$
P^{*}=\frac{\alpha \cdot(1-\alpha) \cdot\left(\tau_{i}-\tau\right) \cdot\left(1-\tau_{i}\right)}{\left\{\tau_{i}+\left[\alpha+\tau_{i} \cdot(1-\alpha)\right] \cdot\left(1-\eta_{\text {reg }}\right) \cdot \frac{Q_{\text {reg }}^{+*}}{Q_{\text {inrev }}^{*}}\right\}}
$$

and the efficiency is:

$$
\eta=\frac{|W|}{Q_{\text {in }}}=\frac{\left|W^{*}\right|}{Q_{\text {inrev }}^{*}+Q_{\text {reg }}^{+^{*}}}=\frac{\left(1-\tau_{i}\right)}{1+\left(1-\eta_{\text {reg }}\right) \cdot \frac{Q_{\text {reg }}^{+^{*}}}{Q_{\text {inrev }}^{*}}} .
$$

$Q_{\text {inrev }}^{*}$ and $\frac{Q_{\text {reg }}^{+*}}{Q_{\text {inrev }}^{*}}$ could be substituted into the above equations with the help of expressions respectively given by Equations (30b) and (37): see Equation (40).

$$
\frac{Q_{\text {reg }}^{+}}{Q_{\text {inrev }}}=\frac{Q_{\text {reg }}^{+^{*}}}{Q_{\text {inrev }}^{*}}=\frac{2 \cdot \gamma \cdot \delta_{p} \cdot B_{V R} \cdot \sqrt{\left[\delta_{p} \cdot \delta_{V R} \cdot \cos \left(\varphi_{V}-\varphi_{p}\right)-1\right]^{2}-\left(\delta_{p}^{2}-1\right) \cdot\left(\delta_{V R}^{2}-1\right)}}{(\gamma-1) \cdot \pi \cdot \varepsilon_{E} \cdot\left[\sqrt{1-\delta_{p}^{2}}-\left(1-\delta_{p}^{2}\right)\right] \cdot \sin \left(\varphi_{p}\right)}
$$




\section{APPLICATIONS OF THE ANALYTICAL SOLUTIONS TO THE PARTIAL CYCLE OPTIMIZATION}

As an example application, the previous equations are used here to describe the influence of the compression to expansion volume ratio $\omega$ and of the speed $n^{*}$ on the main operating parameters $W^{*}, P^{*}$ and $\eta$ (Fig. 3).

For the 3 engine types at the same time, computation and display of these lines and surfaces (and much more), were obtained within few seconds, with Matlab software.

In this first example, the engine is of alpha type, the phase lag angle $\varphi_{0}$ is $\pi / 2$, the gas has a specific heat ratio $\gamma$ of 1.4 , the dead volume ratios $\varepsilon_{E S}, \varepsilon_{C S}, \varepsilon_{R}$ are respectively $0.06,0.06$, 0.08 , the heat conductance ratio $\alpha$ equals 0.5 , the temperature ratio $\tau$ is 0.5 and the regeneratio efficiency $\eta_{\text {reg }}$ is 1 , or 0 (lower right quadrant).

With perfect regeneration, both the work (upper left quadrant) and the efficiency (lower left quadrant) are maximum at very low speed. The work at its overall maximum is obtained for a value of the volume ratio $\omega$ slightly lower than 1 , as previously stated by Walker [6]; the efficiency is constant $(=0.5)$ at $n^{*}=0$ whatever the volume ratio is (basic thermodynamics case). The power representing-surface (upper right quadrant) shows a bended crest of constant height, indicating that maximum power could be obtained for a particular value of $n^{*}$ whatever the volume ratio is, but speed is at a minimum for a value of volume ratio $\omega$ slightly lower than 1. Moreover, a high power could be also obtained within a large range of high speed in a narrow band of low to very low volume ratios, at the cost of high optimum speeds.

Without regeneration (Fig. 3, lower right quadrant), the power is more than halved compared to the value obtained with perfect regeneration and it increases with volume ratio. Moreover, the optimum speed for power is lower, and the band of high power at low volume ratios doesn't really exist.

Another example application concerns the optimization of the power with respect to the phase angle and to the compression-to-expansion volume ratio. With help of Equation (13),
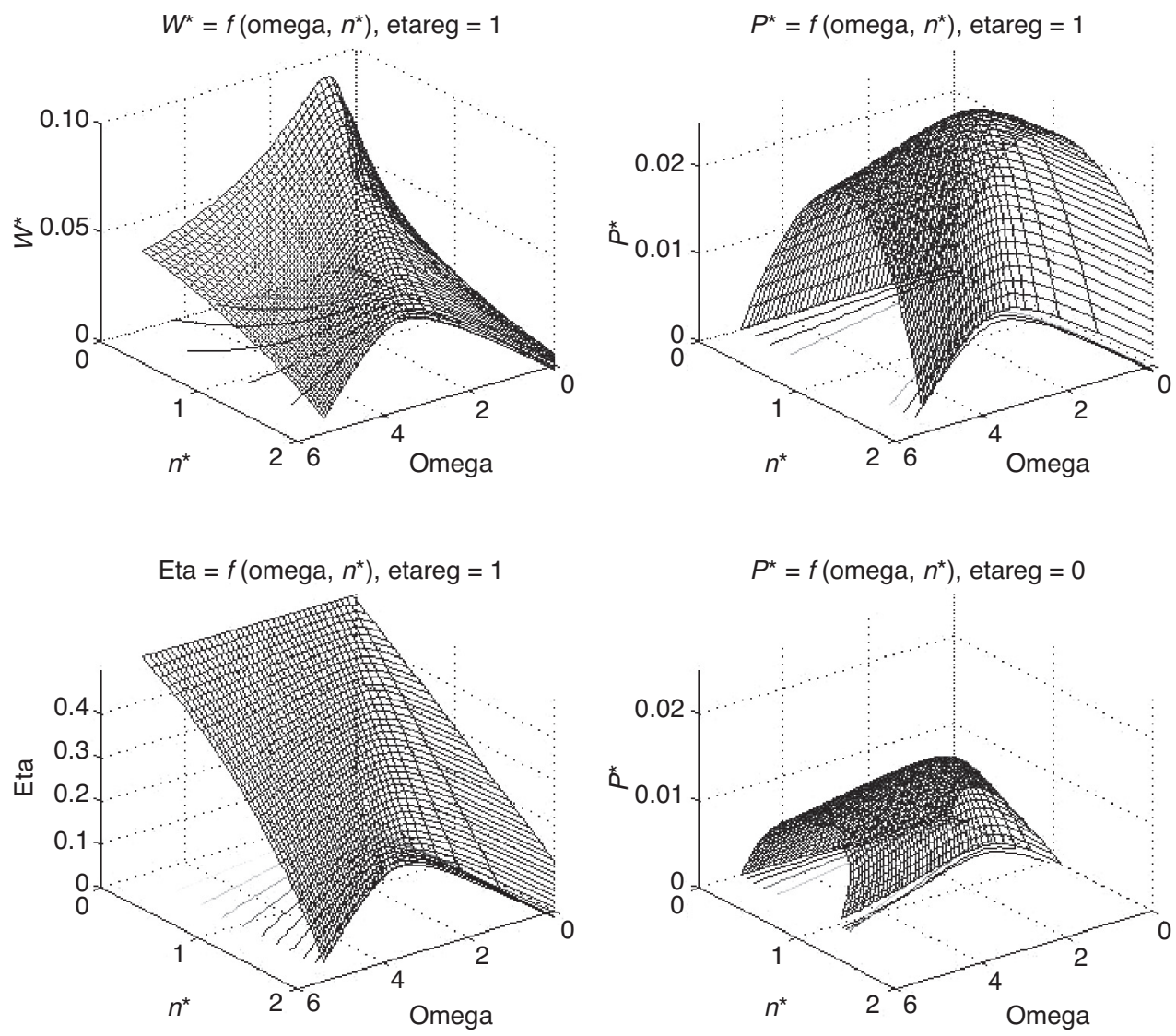

Figure 3

Work, power and efficiency versus volume ratio and speed in case of perfect regeneration and, lower right quadrant, power without regeneration. 

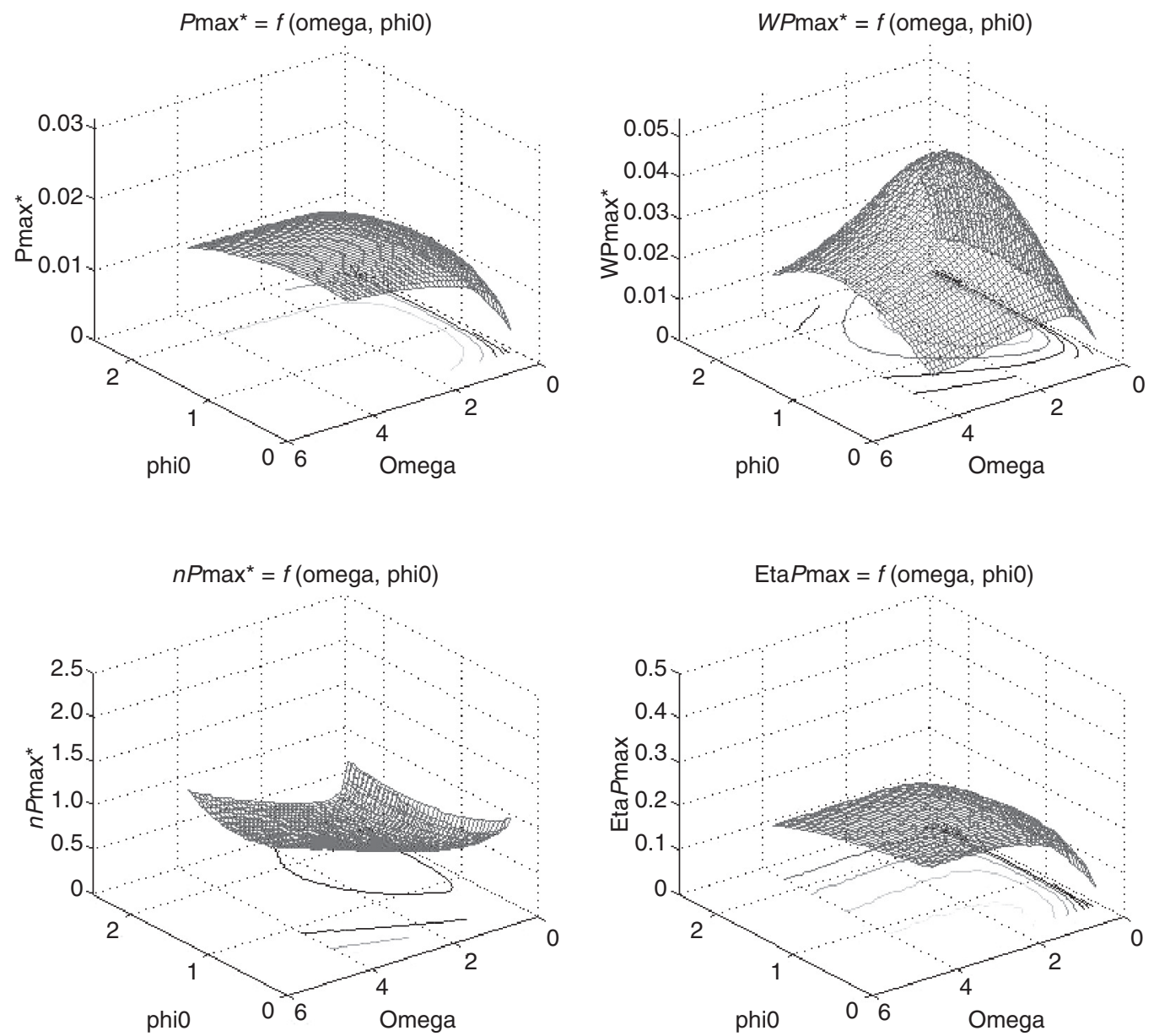

Figure 4

Maximum-power surface and corresponding work, speed and efficiency surfaces versus phase lag angle $\varphi_{0}$ and volume ratio $\omega$.

giving expression of power to be normalized by $K_{T} \cdot T_{H}$, and Equation (40), we get the results illustrated by Figure 4 after few minutes of iterative calculations to find the set of maximum power values and associated values of the other operating parameters. The chosen engine, of which results are displayed here, is of alpha type and the fixed parameters are the same as in the previous example except the regeneration efficiency at 0.5 and the phase lag angle $\varphi_{0}$ considered as a variable. Maximum power increases with $\omega$ increase and $\varphi_{0}$ decrease, but the criterion of maximum power is not the only one to consider as Figure 4 shows: in fact the corresponding work (or torque), the efficiency and the speed of revolution must be examined too. A compromise solution could be found through a high work (or a low speed) or a high efficiency is privileged in addition to maximum power. In this particular case, we see (higher right quadrant) that a maximum work (or torque) is obtained for a phase lag angle approximately equal to 1.6 radian and a volume ratio of approximately 0.8 . This point corresponds nearly to the minimum of speed of revolution (lower left quadrant) with a not-too-much reduced value of efficiency (lower right quadrant). Favoring the efficiency could be done choosing a lower value of phase lag angle and a higher value of volume ratio at the price of a higher speed and lower work (or torque).

These examples show that a first approximate optimization, which, however, neglects conduction- and friction losses, is possible without large efforts.

\section{CONCLUSION}

In this paper, we have studied the exo-irreversible, endoreversible Schmidt-Stirling engine cycle. Analytical expressions were derived for the phase angles at gas flow inversion within the regenerator and for the positive or negative perfectregeneration heat. Adding these ones to other previously 
obtained analytical expressions (expansion and compression volumes, pressure, work, isothermally delivered heat) allowed analytical calculations of each cycle-averaged energy transfer and of efficiency with respect to geometrical and physical parameters (e.g. regenerator efficiency and overall heat conductance) without step-by-step numerical computation of the cycle. An example of cycle optimization with a given phase angle was described; it illustrated the versatility of this set of equations.

Moreover, a second example using this set of equations with an iterative calculation allowed the choice of nearoptimum phase angle and volume ratio to obtain a "good" compromise between maximum power and maximum work (or torque) at minimum speed.

Nevertheless, to be closer to reality, a more elaborate procedure could be followed which takes into account the speed-dependence of physical phenomena such as convective heat transfers, conductive heat losses, gas friction and mechanical friction as, for instance, Senft $[9,10]$ and Petrescu et al. [11] did. Moreover, the normalization could be done with respect to more representative and "absolute" constraint-parameter combinations. For instance, we found earlier [12] that, for an exo-irreversible ideal Stirling cycle, the maximum attainable theoretical work is given by:

$W_{\text {max } \max }=\frac{p_{\max } \cdot V_{T}}{e}(1-\tau)(e=\exp (1) \cong 2.72)$ which could be used instead of $p_{\max } \cdot V_{T}$. It can be established, too, that the maximum heat delivered per unit time (which has dimension of power) is $\dot{Q}_{i n \max \max }=K_{T} \cdot T_{H} \cdot \frac{(1-\tau)}{4}$ which could be used instead of $K_{T} \cdot T_{H}$.

This study could be extended to the exergy balance, to improve the energy use and optimize the cycle by irreversibility localizations (Martaj et al. [13]).

Using this set of equations, a preliminary design of a Stirling engine, to be used in a solar power plant at medium source temperature, is under progress (Nov. 2009).

\section{REFERENCES}

1 Durmayaz A. et al. (2004) Optimization of thermal systems based on finite-time thermodynamics and thermoeconomics, Progr. Energ. Combust. Sci.30, 175-217.

2 Feidt M. et al. (2002) Optimal allocation of Heat Exchanger inventory associated with fixed power output or fixed heat transfer rate input, Int. J. Appl. Thermo. 5, 1, 25-36.

3 Urieli I., Berchowitz D.M. (1984) Stirling cycle engine analysis, Adam Hilger, Bristol, UK.

4 Meijer R.J. (1960) The Philips Stirling thermal engine, $P h D$ Thesis, Delft Technical University.

5 Finkelstein T. (1961) Generalized thermodynamic analysis of Stirling engines (paper 118B) and Optimization of phase angle and volume ratios in Stirling engines (paper 118C), SAE Annual Winter Meeting, Detroit, USA.

6 Walker G. (1973) Stirling-cycle machines, Oxford University Press, Oxford, UK.

7 Rochelle P., Andrzjewski J. (1974) Optimisation des cycles à rendement maximal, Revue de l'Institut Français du Pétrole 29, 731-749.

8 Dwight H.B. (1971) Tables of integrals and other mathematical data, 4th edition, MacMillan Company, New York, USA.

9 Senft J.R. (1998) Theoretical limits on the performance of Stirling engines, Int. J. Energy Res. 22, 991-1000.

10 Senft J.R. (2002) Optimum Stirling engine geometry, Int. J. Energy Res. 26, 1087-1101.

11 Petrescu S., Costea M. et al. (2002) Application of the Direct Method to irreversible Stirling cycles with finite speed, Int. J. Energy Res. 26, 589-609.

12 Grosu L., Rochelle P., Martaj N. (2008) Thermodynamique à échelle finie : optimisation du cycle moteur de Stirling pour l'ingénieur, COFRET'08, June 2008, Nantes, France.

13 Martaj N., Grosu L., Rochelle P. (2007) Thermodynamic study of a low temperature difference Stirling engine at steady state operation, Int. J. Thermo. 10, 4, 165-176.

Final manuscript received in February 2011 Published online in November 2011 


\section{APPENDIX}

$$
\begin{aligned}
& \left\{\begin{array}{l}
a-b \cdot \cos (\varphi)-c \cdot \cos \left(\varphi+\varphi_{0}\right)=B-A \cdot \cos \left(\varphi-\varphi_{I}\right) \\
\text { where } A=\sqrt{b^{2}+c^{2}+2 \cdot b \cdot c \cdot \cos \left(\varphi_{0}\right)}, \quad B=a \\
\cos \left(\varphi_{I}\right)=\frac{b+c \cdot \cos \left(\varphi_{0}\right)}{A}, \quad \sin \left(\varphi_{I}\right)=\frac{c \cdot \sin \left(\varphi_{0}\right)}{A}
\end{array}\right. \\
& p=\frac{m_{T} \cdot r}{\frac{V_{E 0} \cdot[1-\cos (\varphi)]}{2 \cdot T_{h}}+\frac{V_{E 0} \cdot a_{i} \cdot[1+\cos (\varphi)]+V_{C 0} \cdot\left[1-\cos \left(\varphi-\varphi_{0}\right)\right]}{2 \cdot T_{l}}+\frac{V_{R}}{T_{R}}+\frac{V_{E S}}{T_{h}}+\frac{V_{C S}-b_{i} \cdot V_{o l}}{T_{l}}} \\
& D=V_{T} \cdot\left\{\frac{\varepsilon_{E}}{2} \cdot[1-\cos (\varphi)]+\frac{\varepsilon_{E}}{2 \cdot \tau_{i}} \cdot a_{i} \cdot[1+\cos (\varphi)]+\frac{\varepsilon_{C}}{2 \cdot \tau_{i}} \cdot\left[1-\cos \left(\varphi-\varphi_{0}\right)\right]+\varepsilon_{E S}+\left(\varepsilon_{C S}-b_{i} \cdot \varepsilon_{o l}\right) \cdot \frac{1}{\tau_{i}}+\varepsilon_{R} \cdot \frac{T_{h}}{T_{R}}\right\} \\
& \left\{\begin{array}{l}
\oint \frac{\sin (\varphi) \cdot d \varphi}{1-\delta_{p} \cdot \cos \left(\varphi-\varphi_{p}\right)}=\sum_{m=0}^{\infty} \oint \delta_{p}^{m} \cdot\left[\cos \left(\varphi-\varphi_{p}\right)\right]^{m} \cdot \sin (\varphi) \cdot d \varphi \\
=\sum_{m=0}^{\infty} \oint \delta_{p}^{m} \cdot[\cos (\theta)]^{m} \cdot\left[\sin (\theta) \cdot \cos \left(\varphi_{p}\right)+\cos (\theta) \cdot \sin \left(\varphi_{p}\right)\right] \cdot d \theta \\
\text { where } \theta=\varphi-\varphi_{p}, \text { and, then } d \theta=d \varphi
\end{array}\right. \\
& d Q_{\text {reg }}=\frac{V_{R} \cdot d p}{\gamma-1}+\frac{\gamma \cdot r}{\gamma-1} \cdot\left(T_{h} \cdot d m_{E}+T_{l} \cdot d m_{C}\right)=\frac{\gamma}{\gamma-1}\left\{p \cdot\left[d V_{E}^{+}+d V_{C}^{+}\right]+\left[V_{E}^{+}+V_{C}^{+}+\frac{V_{R}}{\gamma}\right] \cdot d p\right\} \\
& {\left[1-\delta_{p} \cdot \cos \left(\varphi-\varphi_{p}\right)\right] \cdot A_{V} \cdot \sin \left(\varphi-\varphi_{V}\right)-\left[B_{V R}-A_{V} \cdot \cos \left(\varphi-\varphi_{V}\right)\right] \cdot \delta_{p} \cdot \sin \left(\varphi-\varphi_{p}\right)=0} \\
& \left\{\begin{array}{l}
\cos \left(\Phi-\Phi^{\prime}\right)=\cos (\Phi) \cdot \frac{\delta_{V R}}{A^{\prime}} \cdot \sin (\Phi)+\sin (\Phi) \cdot \frac{1}{A^{\prime}} \cdot\left[\delta_{p}-\delta_{V R} \cdot \cos (\Phi)\right]=\frac{1}{A^{\prime}} \cdot \delta_{p} \cdot \sin (\Phi) \\
\sin \left(\Phi-\Phi^{\prime}\right)=\left[\frac{\delta_{V R}}{A^{\prime}}-\frac{\delta_{p}}{A^{\prime}} \cdot \cos (\Phi)\right]=\frac{1}{A^{\prime}} \cdot\left[\delta_{V R}-\delta_{p} \cdot \cos (\Phi)\right]
\end{array}\right. \\
& \cos (\theta-\Phi)=\frac{1}{A^{\prime}} \cdot\left\{\delta_{p} \cdot \sin (\Phi) \cdot \cos \left(\theta-\Phi^{\prime}\right)+\left[\delta_{V R}-\delta_{p} \cdot \cos (\Phi)\right] \cdot \sin \left(\theta-\Phi^{\prime}\right)\right\} \\
& Q_{r e g}^{+^{*}}=\frac{\gamma \cdot\left(1-\delta_{p}\right)}{(\gamma-1)} \cdot B_{V R} \\
& \cdot\left[\frac{1-\frac{\delta_{V R} \cdot \delta_{p}}{\delta^{\prime} \cdot A^{\prime}} \cdot\left\{\sin (\Phi)+\sqrt{\delta^{\prime 2}-1} \cdot\left[\frac{\delta_{V R}}{\delta_{p}}-\cos (\Phi)\right]\right\}}{1-\frac{\delta_{p} \cdot \delta_{V R}}{\delta^{\prime} \cdot A^{\prime}} \cdot\left\{\sin (\Phi)-\sqrt{\delta^{\prime 2}-1} \cdot\left[\frac{\delta_{p}}{\delta_{V R}}-\cos (\Phi)\right]\right\}}-\frac{1-\frac{\delta_{V R} \cdot \delta_{p}}{\delta^{\prime} \cdot A^{\prime}} \cdot\left\{\sin (\Phi)-\sqrt{\delta^{\prime 2}-1} \cdot\left[\frac{\delta_{V R}}{\delta_{p}}-\cos (\Phi)\right]\right\}}{1-\frac{\delta_{p} \cdot \delta_{V R}}{\delta^{\prime} \cdot A^{\prime}} \cdot\left\{\sin (\Phi)+\sqrt{\delta^{\prime 2}-1} \cdot\left[\frac{\delta_{p}}{\delta_{V R}}-\cos (\Phi)\right]\right\}}\right]
\end{aligned}
$$


Let $a=1-\frac{\delta_{v R} \cdot \delta_{p}}{\delta^{\prime} \cdot A^{\prime}} \cdot \sin (\Phi)$,

$$
b=\frac{\delta_{V R} \cdot \delta_{p}}{\delta^{\prime} \cdot A^{\prime}} \cdot \sqrt{\delta^{\prime 2}-1} \cdot\left[\cos (\Phi)-\frac{\delta_{V R}}{\delta_{p}}\right] \text { and } c=\frac{\delta_{V R} \cdot \delta_{p}}{\delta^{\prime} \cdot A^{\prime}} \cdot \sqrt{\delta^{\prime 2}-1} \cdot\left[\frac{\delta_{p}}{\delta_{V R}}-\cos (\Phi)\right]
$$

then, $Q_{r e g}^{+*}=2 \cdot \frac{\gamma \cdot\left(1-\delta_{p}\right)}{(\gamma-1)} \cdot B_{V R} \cdot\left[\frac{a \cdot(c-b)}{a^{2}-c^{2}}\right]$; noting that $\frac{\delta_{V R} \cdot \delta_{p}}{\delta^{\prime} \cdot A^{\prime}} \cdot \sin (\Phi)=\frac{B^{\prime}}{\delta^{\prime} \cdot A^{\prime}}=\frac{1}{\delta^{\prime 2}}$,

$\left[\frac{a \cdot(c-b)}{a^{2}-c^{2}}\right]$ is developed as:

$$
\begin{aligned}
& \left(1-\frac{1}{\delta^{\prime 2}}\right) \cdot \frac{1}{\delta^{\prime 2}} \cdot \frac{\sqrt{\delta^{\prime 2}-1}}{\sin (\Phi)} \cdot \frac{\frac{\delta_{V R}}{\delta_{p}}+\frac{\delta_{p}}{\delta_{V R}}-2 \cdot \cos (\Phi)}{\left(1-\frac{1}{\delta^{\prime 2}}\right)^{2}-\left[\frac{1}{\delta^{\prime 2}} \cdot \frac{\sqrt{\delta^{\prime 2}-1}}{\sin (\Phi)}\right]^{2} \cdot\left[\frac{\delta_{p}}{\delta_{V R}}-\cos (\Phi)\right]^{2}}= \\
& \left(1-\frac{1}{\delta^{\prime 2}}\right) \cdot \frac{1}{\delta^{\prime 2}} \cdot \frac{\sqrt{\delta^{\prime 2}-1}}{\sin (\Phi)} \cdot \frac{\frac{A^{\prime 2}}{\delta_{p} \cdot \delta_{V R}}}{\left(1-\frac{1}{\delta^{\prime 2}}\right) \cdot\left[\left(1-\frac{1}{\delta^{\prime 2}}\right)-\frac{\delta_{p}^{2} \cdot\left[\delta_{p}-\delta_{V R} \cdot \cos (\Phi)\right]^{2}}{\left[\delta_{p} \cdot \delta_{V R} \cdot \delta^{\prime} \cdot \sin (\Phi)\right]^{2} \cdot}\right]} \\
& =\left(1-\frac{1}{\delta^{\prime 2}}\right) \cdot \frac{1}{\delta^{\prime 2}} \cdot \frac{\sqrt{\delta^{\prime 2}-1}}{\sin (\Phi)} \cdot \frac{\frac{A^{\prime 2}}{\delta_{p} \cdot \delta_{V R}}}{\left(1-\frac{1}{\delta^{\prime 2}}\right) \cdot\left[\left(1-\frac{1}{\delta^{\prime 2}}\right)-\frac{\delta_{p}^{2} \cdot A^{12} \cdot\left[\sin \left(\Phi^{\prime}\right)\right]^{2}}{A^{\prime 2}}\right]} \\
& =\frac{1}{\delta^{\prime 2}} \cdot \frac{\sqrt{\delta^{\prime 2}-1}}{B^{\prime}} \cdot \frac{A^{\prime 2}}{\left[\left(1-\frac{1}{\delta^{\prime 2}}\right)-\delta_{p}^{2} \cdot\left[1-\cos ^{2}\left(\Phi^{\prime}\right)\right]\right]}=-\frac{\sqrt{\delta^{\prime 2}-1}}{\delta^{\prime}} \cdot \frac{A^{\prime}}{\left\{\left(1-\frac{1}{\delta^{\prime 2}}\right)-\delta_{p}^{2} \cdot\left[1-\frac{1}{\delta^{\prime 2} \cdot \delta_{p}^{2}}\right]\right\}} \\
& =-\frac{\sqrt{\delta^{\prime 2}-1}}{\delta^{\prime}} \cdot \frac{A^{\prime}}{\left(1-\delta_{p}^{2}\right)}=\frac{\sqrt{A^{\prime 2}-B^{\prime 2}}}{\left(1-\delta_{p}^{2}\right)}
\end{aligned}
$$

$$
\frac{Q_{\text {reg }}^{+}}{Q_{\text {inrev }}}=\frac{Q_{\text {reg }}^{+^{*}}}{Q_{\text {inrev }}^{*}}=\frac{2 \cdot \gamma \cdot \delta_{p} \cdot B_{V R} \cdot \sqrt{\left[\delta_{p} \cdot \delta_{V R} \cdot \cos \left(\varphi_{V}-\varphi_{p}\right)-1\right]^{2}-\left(\delta_{p}^{2}-1\right) \cdot\left(\delta_{V R}^{2}-1\right)}}{(\gamma-1) \cdot \pi \cdot \varepsilon_{E} \cdot\left[\sqrt{1-\delta_{p}^{2}}-\left(1-\delta_{p}^{2}\right)\right] \cdot \sin \left(\varphi_{p}\right)}
$$

